\title{
Da Semântica para Demonstrações de Consistência e a Volta
}

\author{
[From Semantics to Consistency Proofs and Back] \\ Rodrigo A. Freire*; e Luiza S. P. Ramos ${ }^{* *}$
}

Resumo: O presente artigo contém duas teses principais. Primeiro, que o ponto de partida em uma demonstração de consistência de um sistema formal é uma noção semântica. Essa tese é apresentada a partir de uma análise das etapas pelas quais uma demonstração de consistência passa, uma vez que um atributo de fórmulas com base em alguma interpretação deve ser estipulado já na etapa inicial. Para avançar cada uma das etapas, uma demonstração de consistência deve produzir um ganho de entendimento correspondente em relação ao sistema. Confirmamos a tese por um estudo de casos de três sistemas em que três demonstrações construtivas de consistência correspondentes são analisadas. O estudo é restrito a demonstrações construtivas, pois no caso modelo-teórico a tese é evidente. Estudamos a lógica de primeira ordem, a aritmética sem indução, também conhecida como aritmética de Robinson, e a aritmética com indução ou de Peano. Em seguida, consideramos a relação entre consistência e verdade na aritmética sob o prisma do estudo de casos. A partir disso, podemos formular a segunda tese: há uma concepção da verdade aritmética que não se compromete de partida com a consistência. Tal tese é motivada tendo em vista as limitações para demonstrações de consistência apresentadas.

Palavras-chave: demonstrações de consistência, aritmética, verdade

Abstract: The current article endorses two main thesis. First we claim that the starting point in a consistency proof of a formal system is a semantic notion. This thesis is supported by an analysis of the stages in which a consistency proof goes through once that already in the initial stage an attribute for formulas must be specified on a interpretative basis. In order to move one stage forward at a time, a consistency proof must give an additional information corresponding to the system. We corroborate the thesis with case studies of three systems by which their constructive consistency proofs are analysed. Each study is restricted to constructive proofs since in the model-theoretic case the thesis is trivial. We analyse first-order logic, arithmetic without induction, also known as Robinson's arithmetic, and arithmetic with induction or Peano's. Then, we consider the relation between consistency and truth in Peano arithmetic in light of the case study. In regard to this we can formulate our second thesis: there is a conception of arithmetic truth in which no commitment with consistency must be taken for granted. The motivation for this thesis is the limitation for achieving constructive consistency proofs presented in the article. Keywords: consistency proofs, arithmetics, truth

${ }^{*}$ Professor do Departamento de Filosofia da Universidade de Brasília (UnB). E-mail: freirester@gmail.com.

${ }^{* *}$ Mestranda no PPGM da UnB. E-mail: luizaspramos@gmail.com. 


\section{1 - Considerações Gerais Sobre Demonstrações de Consistência}

Partimos da noção de sistema formal, estabelecida a partir das investigações sobre os fundamentos da matemática que ocorreram no final do século XIX e no início do século XX. O problema da demonstração de consistência surge no contexto de tais investigações, principalmente a partir da desinterpretação dos sistemas axiomáticos. Para um estudo do desenvolvimento dos sistemas formais veja Arno e Raggio. Para mais detalhes técnicos e históricos veja Bourbaki, especialmente a Note Historique. A consistência de teorias matemáticas se torna objeto de um estudo matemático apenas a partir de tal desenvolvimento, como resume Kleene:

É uma contribuição de Hilbert ter concebido uma nova abordagem direta, e ter reconhecido o que esta envolvia para a axiomatização. Este método direto está implícito no significado de consistência (pelo menos como agora o pensamos), nomeada- mente que uma contradição lógica ( uma proposição $A$ e sua negação $\neg A$ serem ambas teoremas) não pode surgir da teoria deduzida a partir dos axiomas. Portanto, para demonstrar a consistência de uma teoria diretamente, deve-se demonstrar uma proposição sobre a teoria ela própria, isto é, especificamente sobre todas as possíveis demonstrações de teoremas na teoria. A teoria matemática cuja consistência queremos demonstrar tornase assim ela própria objeto de um estudo matemático, o qual Hilbert denomina "metamatemática" ou "teoria da demonstração". ${ }^{1}$

Usamos como sinônimas as expressões 'sistema formal', 'sistema axiomático formalizado' e 'sistema dedutivo'. Demonstrar a consistência de um sistema formal para uma teoria clássica é demonstrar que nem toda fórmula do sistema é dedutível no sistema. Estamos interessados em elucidar a estrutura das demonstrações de

\footnotetext{
${ }^{1}$ Tradução nossa do original, It is Hilbert's contribution now to have conceived a new direct approach, and to have recognized what it involves for the axiomatization. This direct method is implicit in the meaning of consistency (at least as we now think of it), namely that no logical contradiction (a proposition A and its negation not A both being theorems) can arise in the theory deduced from the axioms. Thus to prove consistency of a theory directly, one should prove a proposition about the theory itself, i.e. specifically about all possible proofs of theorems in the theory. The mathematical theory whose consistency it is hoped to be proved then becomes itself the object of a mathematical study, which Hilbert calls "metamathematics" or "proof theory". (Kleene, pg.55.)
} 
consistência, entender quais são os problemas que devemos resolver para alcançar tais demonstrações, avaliar as dificuldades impostas por esses problemas e seu impacto para a fundamentação da matemática.

Vamos começar pela apresentação de um esquema das etapas que compõem, em geral, tais demonstrações. Podemos destacar quatro etapas que constituem um esquema geral para entender demonstrações de consistência. Enumeramos abaixo essas quatro etapas, explicadas uma a uma a seguir.

1. Uma propriedade $\mathcal{P}$ de fórmulas do sistema é escolhida.

2. Uma classe $\Delta$ de fórmulas do sistema é determinada.

3. A correção com relação a $\mathcal{P}$ das fórmulas em $\Delta$ que são dedutíveis é estabelecida.

4. Uma fórmula em $\Delta$ que não satisfaz $\mathcal{P}$ é determinada.

A propriedade $\mathcal{P}$ de fórmulas do sistema deve ser escolhida criteriosamente, o sucesso das etapas seguintes depende de uma escolha apropriada no início. As escolhas apropriadas de $\mathcal{P}$ são, em geral, tais que se $A$ é uma fórmula do sistema, então $\mathcal{P}(A) \mathrm{diz}$ que $A$ é o caso segundo uma interpretação definida. Nas demonstrações modelo-teóricas de consis- tência $\mathcal{P}(A)$ diz que $A$ é válida em uma estrutura determinada. De forma similar, nas demonstrações construtivas partimos de um entendimento semântico, no entanto buscamos extrair uma propriedade construtiva, ou até mesmo finitária, desse entendimento. Reforçamos que o ponto de partida das demonstrações de consistência, o que guia a escolha da propriedade $\mathcal{P}$, mesmo no caso das demonstrações finitárias, é semântico, o que é explicado de modo claro por Kleene:

Todas essas demonstrações de consistência dependem da disponibilidade de um modelo para os axiomas, assim como aquelas dadas antes do advento da teoria de Hilbert da demonstração. Mas dar um modelo para os axiomas em termos de uma aritmética intuitiva não estabelece, além de toda dúvida, que nenhuma contradição pode surgir na teoria que e deduzida a partir dos axiomas, a não ser que possa também ser demonstrado que os argumentos na teoria possam ser traduzidos em argumentos aritméticos intuitivos nos termos dos objetos usados no mo- 
delo. ${ }^{2}$

Essa passagem também chama a atenção para a importância das etapas seguintes, especialmente do que estamos chamando de correção. Não basta escolher a propriedade $\mathcal{P}$. Há uma correspondência direta entre (i) dar um modelo em termos de uma aritmética intuitiva e demonstrar que os argumentos da teoria possam ser traduzidos em argumentos aritméticos intuitivos nos termos dos objetos usados no modelo, conforme a formulação de Kleene, e (ii) dar uma propriedade finitária $\mathcal{P}$ e demonstrar a correção com relação a essa propriedade das fórmulas dedutíveis, ou pelo menos parte delas, de acordo com nosso esquema.

Por isso, as segundas e terceiras etapas têm por finalidade isolar uma classe $\Delta$ de fórmulas suficientemente amplas e tal que toda fórmula em $\Delta$ que é dedutível no sistema cuja consistência está em estudo satisfaz $\mathcal{P}$. A classe $\Delta$ não precisa ser a classe de todas as fórmulas do sistema, mas precisa conter alguma fórmula para que a última etapa seja possível. $\mathrm{Na}$ etapa final determina-se uma fór- mula em $\Delta$ que não satisfaz $\mathcal{P}$. Pela etapa anterior, tal fórmula também não é dedutível e, portanto, o sistema é consistente. Assim, o núcleo de uma demonstração construtiva de consistência é composto pela escolha de uma propriedade $\mathcal{P}$ que seja construtiva e pela demonstração construtiva da correção com relação a $\mathcal{P}$ de uma classe apropriada de fórmulas dedutíveis.

\section{2 - Por Que Buscar Demonstra- ções Construtivas de Consistên- cia}

Como já ressaltamos, o problema da consistência está bem formulado, pelo menos a partir de Hilbert, com base na possibilidade de representar a matemática sem recurso ao infinito. ${ }^{3}$ A tentativa de resolver esse problema preliminar é baseada na ideia que bastaria representar o uso dos símbolos, e isso pode ser feito por meio de regras de uso que são, também, de natureza simbólica. Qualquer representação de entendimento de outra natureza do simbolismo não seria requerida. Essa possibilidade pode ser vislumbrada obser-

\footnotetext{
${ }^{2}$ Tradução nossa do original, These consistency proofs all depend on having a model for the axioms, as did those given before the advent of Hilbert's proof theory. But giving a model for the axioms in intuitive arithmetical terms does not establish beyond all doubt that no contradiction can arise in the theory deduced from the axioms, unless it can also be demonstrated that the reasonings in the theory can be translated into intuitive arithmetical reasonings in terms of the objects used in the model. (Kleene, p. 475.)

${ }^{3}$ Veja Hilbert, especialmente as páginas 191 e 192.
} 
vando que o pensamento matemático opera por meio de frases na sua atividade de definir e demonstrar. Definições são determinadas por frases descritivas e demonstrações por encadeamentos de frases. O caminho para representar de modo puramente simbólico a atividade matemática passaria, então, pela construção de um sistema de frases com a delimitação precisa das suas regras de uso.

O emprego das frases nas deduções do sistema é determinado pela estipulação dos axiomas, ou seja, de quais frases podem ser usadas como premissas, e das regras de inferência, que determinam quais passagens são válidas na atividade dedutiva. Também é necessário saber usar frases descritivas para introduzir novos símbolos a partir dos símbolos inicialmente estipulados. Um símbolo como ' $\pi$ ' seria introduzido no nosso sistema que representa a matemática com uma descrição que determina de modo completo, dentro do sistema, o uso do novo símbolo. Note que o significado do símbolo ' $\pi$ ' não é parte constituinte desse processo e o infinito não ocorre nessas estipulações.

A lógica de primeira ordem serve de base para uma representação livre de infinitude atual da atividade matemática a partir de seu sistema dedutivo de frases e regras simbólicas. Para que o sis- tema dedutivo da lógica de primeira ordem desempenhe o papel esperado não é aceitável usar o infinito em lugar algum no processo de constituição do mesmo e tampouco no correspondente processo de redução do raciocínio matemático normal aos procedimentos simbólicos desse veículo formal. Ou seja, constituir o sistema finitariamente é apenas o primeiro passo. Devemos também mostrar que o sistema funciona adotando a mesma restrição metodológica sobre o infinito. Para isso, há que se mostrar que as passagens do raciocínio matemático em geral estão representadas no sistema e ao mesmo tempo que o sistema não é capaz de deduzir frases além da conta. Essa última propriedade está fortemente ligada à possibilidade de demonstrar construtivamente a consistência do sistema.

$O$ que seria dizer de um sistema de representação simbólica da matemática que ele não deduz frases além da conta? Uma primeira resposta seria que para a classe das frases do sistema que admitem de modo uniforme um significado finitário há uma demonstração construtiva que se uma frase dessa classe é deduzida no sistema, então seu significado finitário é verdadeiro, caso em que dizemos que o sistema é finitariamente correto com relação 
à classe. Uma análise nesse sentido é apresentada a seguir.

Vamos supor que $M$ é uma imagem formal de parte da matemática, um sistema simbólico tal que parte das passagens do raciocínio matemático estão representadas em $M$. Sabemos que $M$ é consistente se e somente se há uma frase do sistema que não é dedutível. Outra caracterização da consistência de $M$ é que não há frase $A$ do sistema tal que $A$ é dedutível em $M$ e $\neg A$ também é dedutível em $M$, pois qualquer frase é consequência tautológica de $A$ e $\neg A$. Para cada frase $A$, não pode ser que ambas $A$ e $\neg A$ estejam na conta do que é para ser deduzido. De todo modo, fica claro que se $M$ é inconsistente, então $M$ é capaz de deduzir frases além da conta. Isso é importante, mas seria a conversa válida? Será que bastaria que $M$ fosse consistente para garantir que $M$ não é capaz de deduzir o que não é para ser deduzido? A resposta aqui é não. O sistema pode ser consistente e, por exemplo, deduzir uma sentença $A$ que é interpretada finitariamente como falsa. A mera consistência de $M$ não basta.

Uma hipótese mais forte que a mera consistência de $M$ é a hipótese que há uma demonstração construtiva da consistência de $M$.
Seria isso ainda insuficiente? A resposta agora depende do que entendemos como aquilo que não é para ser deduzido. Entre as sentenças de $M$ há a classe daquelas que admitem uniformemente um significado finitário. Agora, qual é essa classe? Vamos estipular, por enquanto, que é a classe das sentenças $\forall$-rudimentares (ver Boolos, p. 262, e Smorynski, p. 823). Tal estipulação implica que a hipótese acima é suficiente, conforme o teorema abaixo. ${ }^{4}$

Suponha que $M$ representa parte suficiente da matemática e que há uma demonstração construtiva da consistência de $M$. Nesse caso, $M$ é finitariamente correto com relação à classe das sentenças $\forall$-rudimentares.

A classe das sentenças $\forall$ rudimentares é tal que suas sentenças admitem uniformemente um significado finitário. Se $A$ é uma sentença $\forall$-rudimentar, representamos por $\tilde{A}$ o enunciado finitário correspondente. Seja $A$ uma sentença $\forall$-rudimentar dedutível em $M$. Então, há uma demonstração construtiva que $A$ é dedutível em $M$, que consiste em exibir a dedução de $A$ em $M$. Por outro lado, $\neg A$ é uma sentença $\exists$ rudimentar, e há uma demonstração construtiva que se $\neg A$ não é dedutível em $M$, então $\tilde{A}$ (ver Bo-

${ }^{4} \mathrm{O}$ resultado encontra-se também em Smorynski, p. 824. 
olos p.267). Pela hipótese do teorema, há uma demonstração construtiva que pelo menos uma entre $A$ e $\neg A$ não é dedutível em $M$. Portanto, há uma demonstração construtiva de $\tilde{A}$.

O teorema acima não pode ser ampliado para abarcar a classe das sentenças $\exists$-rudimentares. De fato, considere o sistema $Q$ da aritmética minimal definido em Boolos, p. 266. Há uma demonstração construtiva, e até mesmo finitária, ${ }^{5}$ que se $Q$ é consistente, então a sentença de Gödel para $Q$ não é dedutível em $Q$. Isso é parte da demonstração do primeiro teorema de Gödel da incompletude (ver Boolos, p. 288). Há também uma demonstração finitária da consistência de $Q$, como veremos, logo da não-dedutibilidade de $G$ em Q. Há ainda, apenas com métodos finitários, uma demonstração que se $G$ não é dedutível em $Q$, então o sistema $Q[\neg G]$, obtido a partir de $Q$ pela adição de $\neg G$ como axioma, é consistente. Portanto, há uma demonstração finitária da consistência de $Q[\neg G]$. Como o enunciado finitário $\tilde{G}$ correspondente a $G \operatorname{diz}$ que $G$ não é dedutível em $Q$, o enunciado finitário correspondente a $\neg G$ diz que $G$ é dedutível em $Q$. Concluímos que $\neg G$ não é finitariamente correta, apesar de haver uma demonstração finitária da consistência do sistema $Q[\neg G]$ onde $\neg G$ é dedutível. Como $\neg G$ neste contraexemplo é $\exists$-rudimentar, o teorema acima não se aplica a essa classe de sentenças.

Portanto, uma demonstração construtiva de consistência é suficiente para legitimar o uso de $M$ para demonstrar sentenças $\forall$ rudimentares, mas insuficiente para o uso correspondente às $\exists$ rudimentares. Contudo, há outras consequências interessantes de uma demonstração construtiva de consistência. Uma dessas consequências é que, para demonstrar construtivamente a consistência de um sistema é preciso entender a estrutura fina do funcionamento do sistema. Vamos tentar explicar isso em termos do esquema de quatro etapas da seção anterior.

Uma demonstração construtiva de consistência de um sistema $M$ que segue as quatro etapas listadas deve apresentar uma construção que mostra como a propriedade $\mathcal{P}$ se aplica às fórmulas dedutíveis em $M$ que estão na classe $\Delta$. Além disso, uma tal demonstração deve mostrar uma fórmula em $\Delta$ para a qual a propriedade construtiva $\mathcal{P}$ não se aplica. Como

\footnotetext{
${ }^{5}$ A distinção entre demonstração finitária e demonstração meramente construtiva que adotamos aqui é explicada em Shoenfield, p. 214.
} 
já foi dito, a escolha de uma propriedade $\mathcal{P}$ adequada para essa construção demanda um entendimento construtivo da semântica de $M$ com as fórmulas dedutíveis em $M$ que estão na classe $\Delta$, ou seja, um entendimento de que tipo de propriedade construtiva se aplica a elas. Vamos ilustrar o ponto com um exemplo simples na seção seguinte.

\section{3 - A Demonstração da Consis- tência da Lógica de Primeira Or- dem}

Considere $M$ um sistema dedutivo para a lógica clássica de primeira ordem sem igualdade. É possível demonstrar finitariamente que $M$ é consistente, ou seja que alguma fórmula não é dedutível em $M$. A ideia da demonstração é fácil de explicar nesse caso. Basta observar que as fórmulas dedutíveis em $M$ são válidas em todas as estruturas não-vazias. Em particular as fórmulas dedutíveis em $M$ são válidas nas estruturas com um único indivíduo no domínio, e a validade em todas as estruturas com um único indivíduo no domínio pode ser caracterizada como uma propriedade finitária. Vejamos como essa ideia pode ser transformada em uma demonstração.

Seja $A$ uma fórmula qualquer e $b$ um nome próprio novo, e seja $A^{*}$ a fórmula obtida a partir de $A$ pela omissão de todos os quantificadores e substituição de todos os termos restantes por $b$. Se $A$ é dedutível a partir de $M$, então $A^{*}$ é uma tautologia.

Se $A$ é dedutível em $M$, então $A$ é um axioma do tipo $B_{x}[a] \rightarrow \exists x B$, ou é consequência tautológica de fórmulas dedutíveis, ou é do tipo $\exists x B \rightarrow C$, em que $x$ não ocorre livre em $C$ e $B \rightarrow C$ é dedutível. Podemos demonstrar por indução que $A^{*}$ é uma tautologia em qualquer dos três casos acima.

Primeiro, o passo base. Se $A$ é um axioma do tipo $B_{x}[a] \rightarrow \exists x B$, então $A^{*}$ é $B^{*} \rightarrow B^{*}$, e isso é uma tautologia. Agora o passo indutivo. Se $A$ é consequência tautológica de fórmulas dedutíveis $B_{1}, \ldots$, $B_{n}$, então $A^{*}$ é consequência tautológica de $B_{1}^{*}, \ldots, B_{n}^{*}$. Por hipótese de indução, $B_{1}^{*}, \ldots, B_{n}^{*}$ são tautologias, de onde segue que $A^{*}$ é tautologia. Por outro lado, suponha que $A$ é $\exists x B \rightarrow C$, em que $x$ não ocorre livre em $C$ e $B \rightarrow C$ é dedutível. Nesse caso, $A^{*}$ é $B^{*} \rightarrow C^{*}$, e é tautologia por hipótese de indução.

A consistência do sistema $M$ é consequência do teorema acima. De fato, a fórmula $B \wedge \neg B$ não é dedutível pois $(B \wedge \neg B)^{*}$ é $B^{*} \wedge \neg B^{*}$, que não é uma tautologia. Temos, portanto, uma demonstração finitária da consistência da lógica de primeira ordem. Vamos analisar 
esta demonstração em detalhes.

Primeiro, identificamos a propriedade finitária $\mathcal{P}$. Afirmar que $A$ satisfaz $\mathcal{P}$ é afirmar que sua transformada $A^{*}$ é uma tautologia. Observamos que, assim definida, $\mathcal{P}$ é uma propriedade finitária equivalente à validade em todas as estruturas com um único indivíduo no domínio. Vamos demonstrar, por indução na complexidade de $A$, que $A$ é satisfeita em qualquer estrutura com um único indivíduo se e somente se $A^{*}$ é uma tautologia, ou seja, verdadeira segundo o método usual das tabelas de verdade para qualquer atribuição de valor de verdade para suas subfórmulas atômicas.

Sejam $p_{1}, \ldots, p_{m}$ símbolos de predicado e $\mathcal{N}$ uma estrutura com um único indivíduo $\alpha$ e que interpreta esses símbolos. Há exatamente duas opções para a interpretação de cada símbolo de predicado $p_{i}$, com $1 \leq i \leq m: \mathrm{Ou}$ a interpretação de $p_{i}$ é a extensão vazia, ou é a extensão total $\{\langle\alpha, \ldots, \alpha\rangle\}$, determinada pela aridade de $p_{i}$, correspondendo naturalmente é atribuição de falso ou de verdadeiro. Desse modo, a estrutura $\mathcal{N}$ corresponde a uma linha da tabela de verdade para $m$ símbolos proposicionais.

Com isso, para qualquer fórmula $A$ cujos símbolos de predicado estão entre $p_{1}, \ldots, p_{m}$, temos que $A$ é satisfeita em $\mathcal{N}$ se e somente se $A^{*}$ é verdadeira na linha da tabela de verdade correspondente a $\mathcal{N}$. Se $A$ é atômica, da forma $p_{i}(t, u, v, \ldots)$, então $A^{*}$ é $p_{i}(b, b, b, \ldots)$. Se a interpretação de $p_{i}$ é a extensão total, então, como $b$ e $t, u, v, \ldots$ denotam $\alpha$, tanto $A$ quanto $A^{*}$ são satisfeitas em $\mathcal{N}$ e $A^{*}$ é verdadeira na linha correspondente. Se a interpretação de $p_{i}$ é a extensão vazia, então $A$ e $A^{*}$ não são satisfeitas e $A^{*}$ é falsa na linha correspondente. Se $A$ é $\neg B$ ou é $B \vee C$, então $A^{*}$ é $\neg B^{*}$ ou é $B^{*} \vee C^{*}$ e o resultado segue da hipótese de indução nesses casos. Finalmente, se $A$ é $\exists x B$, então $A^{*}$ é $B^{*}$. Agora, $A$ é satisfeita em $\mathcal{N}$ com seus termos denotando $\alpha$ se e somente se $B$ é satisfeita em $\mathcal{N}$ quando seus termos denotam $\alpha$. Por hipótese de indução, $B$ é satisfeita em $\mathcal{N}$ quando seus termos denotam $\alpha$ se e somente se $B^{*}$ é verdadeira na linha correspondente a $\mathcal{N}$, e o resultado segue do fato que $A^{*}$ é $B^{*}$.

Concluímos, a partir do parágrafo acima, que $A$ é válida em todas as estruturas apropriadas com um único indivíduo se e somente se $A^{*}$ é uma tautologia. Tal fato não é usado na demonstração de consistência, mas explica de onde surge a ideia subjacente. É importante se perguntar por que usamos a propriedade $\mathcal{P}$, "a transformada é uma tautologia”, no lugar da propriedade "ser válida nas 
estruturas com um único indivíduo". Acontece que a noção geral de validade em estruturas não é finitária, por exemplo, a cláusula sobre a interpretação da quantificação existencial em uma estrutura não é finitária. Por isso, precisamos primeiro eliminar o elemento infinitário, a interpretação da quantificação, para apresentar a propriedade $\mathcal{P}$ no âmbito finitário. Sem a eliminação dos quantificadores isso não seria possível. Argumentamos que trata-se de um padrão: é necessário eliminar a quantificação para realizar as etapas de uma demonstração construtiva de consistência.

\section{4 - A Consistência da Aritmética de Primeira Ordem}

As etapas das demonstrações de consistência que analisaremos aqui também são articuladas a partir de uma noção de validade. Tal noção deve por um lado ser adequadamente restrita para admitir uma caracterização finitária ou pelo menos construtiva, e por outro lado ampla o suficiente para acomodar a correção de uma classe apropriada de fórmulas.

Vamos começar analisando a demonstração da consistência de um sistema para a aritmética sem o axioma esquema da indução, o sistema $N$ apresentado em Shoenfield, página 22. Trata-se de uma teoria aberta, ou seja, tal que os axiomas não-lógicos são livres de quantificadores. Nesse caso, como consequência do primeiro teorema epsilon ${ }^{6}$, se uma fórmula $A$ livre de quantificadores é dedutível em $N$ então $A$ é consequência tautológica de instâncias de axiomas também livres de quantificadores de N. A partir desse resultado, uma demonstração finitária da consistência de $N$ pode ser obtida e inserida nas quatro etapas do nosso esquema.

Primeiro, considere $\mathcal{P}$ a propriedade de validade finitária definida como: Uma fórmula $A$ é finitariamente válida se e somente se o valor de verdade de qualquer instância fechada de $A$ é computável e verdadeiro. Dizemos que o valor de verdade de qualquer instância fechada de $A$ é computável e verdadeiro quando o procedimento uniforme que calcula o valor de verdade de uma dada instância de $A$ segundo as tabelas de verdade para os conectivos lógicos e os algoritmos para sucessor, soma, produto, relação de igualdade e relação de ordem dá como resultado o valor verdadeiro. $\mathrm{Ob}$ servamos que os axiomas abertos de $N$, o que inclui os axiomas nãológicos e os axiomas da igualdade,

\footnotetext{
${ }^{6}$ Veja a terceira seção do verbete https://plato.stanford.edu/entries/epsilon-calculus/
} 
são finitariamente válidos.

Segundo, seja $\Delta$ a classe das fórmulas abertas, ou seja, livres de quantificadores. Como vimos acima, se uma fórmula $A$ em $\Delta$ é dedutível em $N$, então $A$ é consequência tautológica de instâncias de axiomas abertos de $N$. Mas uma consequência tautológica de fórmulas finitariamente válidas é também finitariamente válida. As instâncias dos axiomas abertos de $N$ são finitariamente válidas, $\mathrm{e}$ disso segue que as fórmulas abertas dedutíveis em $N$ são todas finitariamente válidas. Ou seja, que vale a correção com relação a $\mathcal{P}$ das fórmulas em $\Delta$ que são dedutíveis em $N$. Finalmente, a fórmula aberta $\neg x=x$ não é finitariamente válida, portanto não é dedutível em $N$, o que mostra sua consistência.

A demonstração acima tem dois pontos cruciais. O primeiro é que há um procedimento uniforme, com base nas tabelas de verdade e nos algoritmos para as operações e relações aritméticas básicas, para calcular o valor de verdade de qualquer sentença livre de quantificadores escrita na linguagem de $N$. O segundo, é o uso do primeiro teorema epsilon, que estabelece a correção com relação a $\mathcal{P}$ das fórmulas em $\Delta$ que são dedutíveis em $N$. A demonstração deste teorema é a parte mais difícil, que consiste em eliminar quantificadores. Notamos que a possibilidade de eliminar a quantificação é o elemento comum de ambos os pontos, sem o qual não alcançaríamos a propriedade de validade finitária ou a correção.

Passemos ao caso da aritmética de primeira ordem com indução. Novamente seguimos a referência Shoenfield, em que a aritmética com indução é apresentada na página 204. Vamos denotar esse sistema por $P A$. Como o axioma esquema da indução não é livre de quantificadores, os axiomas de $P A$ não são finitariamente válidos no sentido acima. Para demonstrar a consistência de $P A$ é preciso apresentar uma propriedade $\mathcal{P}$, demonstrar a correção com relação a $\mathcal{P}$ das fórmulas em uma classe $\Delta$ dedutíveis em $P A$, e encontrar uma fórmula em $\Delta$ que não satisfaz $\mathcal{P}$. Para uma demonstração finitária, a propriedade $\mathcal{P}$ deveria ser finitária, assim como a demonstração da correção das fórmulas em $\Delta$ dedutíveis e da incorreção de alguma fórmula em $\Delta$. Infelizmente, o segundo teorema de Gödel da incompletude faz parecer duvidoso que isso seja possível. Contudo, há demonstrações de consistência da aritmética de primeira ordem com indução que vão pouco além do finitário. A mais conhecida é devida a Gentzen, mas o próprio Gödel produziu uma demonstração 
assim, que foi apresentada como uma reformulação da demonstração de Gentzen.

Vamos analisar uma variante da demonstração de Gödel da consistência de $P A$ devida a Shoenfield, que apresenta uma interpretação do sistema $P A$ para a aritmética com indução diretamente em uma teoria de funcionais recursivos de tipo finito. ${ }^{7}$ A linguagem da teoria não tem quantificadores, mas tem uma quantidade enumerável de tipos, e estoques de pronomes para cada um desses tipos. A definição dos termos e constantes (símbolos para funcionais) é indutiva, de forma a dar esquemas de introdução de constantes a partir das constantes básicas 0 e $\mathrm{S}$ (intencionalmente "zero" e "sucessor") e da descrição dos tipos. Além de esquemas puramente combinatórios, há um esquema de introdução de símbolos para funcionais por recursão. As fórmulas atômicas são equações apenas entre termos de tipo $o$, que intencionalmente se referem a números. As demais fórmulas são obtidas por negações e disjunções apenas.

A interpretação de $P A$ é então definida associando para cada fórmula $A$ do sistema uma fórmula transformada $A^{*}$ do tipo $\forall \bar{x} \exists \bar{y} \phi$, em que $\phi$ é uma fórmula da te- oria descrita acima e $\bar{x}$ e $\bar{y}$ são sequências de pronomes. Exatamente como no caso da demonstração da consistência da lógica de primeira ordem, uma fórmula $A$ e sua transformada $A^{*}$ possuem o mesmo significado pretendido, o que não é usado na demonstração. No próximo passo, um teorema de correção é demonstrado relacionando a demonstrabilidade de $A$ com a validade da respectiva fórmula transformada $A^{*}$ na teoria dos funcionais. A demonstração do teorema de correção é construtiva, e dele segue o resultado de consistência.

O único elemento não-finitário da demonstração de Gödel da consistência de $P A$ é a noção de validade da teoria dos funcionais. Mas essa noção não está tão distante do finitário quanto, por exemplo, a noção de validade padrão da aritmética de primeira ordem, esta sim fortemente nãofinitária. O motivo para isso é que não há fórmulas quantificadas na teoria dos funcionais, portanto a noção de validade associada não inclui cláusulas para quantificadores. Na verdade, a única cláusula relevante é aquela que corresponde às fórmulas atômicas, equações entre termos de tipo $o$. A semântica dessas equações não

\footnotetext{
${ }^{7}$ Vamos apresentar apenas um breve esboço da teoria de funcionais e da demonstração de consistência suficiente para nossos propósitos. Para mais detalhes recomendamos Shoenfield, pp. 214 - 222.
} 
é finitária porque símbolos para funcionais de tipo superior podem ocorrer nos termos de tipo $o$, o que torna a interpretação do termo geral de tipo $o$ não-finitária. Tal elemento semântico está presente na demonstração de consistência e parece que não pode ser removido. Por outro lado, as relações entre funcionais recursivos de tipo finito parecem suficientemente determinadas e, como a semântica do termo geral de tipo $o$ não vai muito além do âmbito finitário, a consistência de $P A$ baseada na demonstração com teoria dos funcionais é plausível.

A demonstração que acabamos de descrever é facilmente colocada no esquema geral de demonstrações de consistência. Primeiro, dizemos que uma fórmula $A$ de $P A$ tem a propriedade $\mathcal{P}$ se a fórmula $A^{*}$ correspondente, que é do tipo $\forall \bar{x} \exists \bar{y} \phi$, é válida no sentido que há um procedimento finitário e uniforme que produz termos $\bar{y}$ a partir de termos $\bar{x}$ de modo que a instância associada de $\phi$ seja válida no sentido da teoria dos funcionais. Depois, tomando como $\Delta$ o conjunto de todas as fórmula, demonstramos finitariamente a correção com relação é $\mathcal{P}$ de todas as fórmulas dedutíveis. Por fim, demonstramos que a fórmula $\neg 0=0$ não possui a propriedade $\mathcal{P}$. De todas essas etapas, apenas a definição da propriedade
$\mathcal{P}$ apresenta algum elemento nãofinitário.

Podemos dizer que essa demonstração é construtiva apesar de não ser estritamente finitária. Novamente, temos aqui dois pontos cruciais. O primeiro é o estabelecimento da teoria dos funcionais e da noção de validade nessa teoria, a partir da qual a propriedade $\mathcal{P}$ é definida. O segundo é demonstrar a correção com relação a $\mathcal{P}$ das fórmulas são dedutíveis em $P A$. Para isto é preciso mostrar, com um procedimento finitário e uniforme, como produzir para toda fórmula dedutível $A$ termos apropriados a partir de termos dados, de modo que a instância associada da matriz da fórmula transformada $A^{*}$ seja válida no sentido da teoria dos funcionais. Notamos também que a eliminação da quantificação está presente em cada parte da demonstração pois sem isso não alcançaríamos a propriedade "quase-finitária" $\mathcal{P}$ ou a correção.

A partir de nossa análise podemos destacar como elemento comum nas demonstrações de consistência tanto a eliminação de quantificadores como a observação de que a propriedade $\mathcal{P}$ é derivada de uma semântica padrão. Os dois pontos estão relacionados e não sem razão. Uma vez que uma demonstração de consistência é obtida a partir da correção de 
algumas fórmulas dedutíveis com relação a $\mathcal{P}$, é natural derivar a propriedade $\mathcal{P}$ de uma semântica padrão para a qual já temos correção. Contudo a principal componente não-finitária de uma semântica padrão é a cláusula correspondente à quantificação. Portanto, para derivar de uma semântica padrão uma propriedade $\mathcal{P}$ que seja pelo menos próxima do âmbito finitário, é preciso eliminar quantificadores. No caso de $P A$ isso aparentemente não pode ser feito sem custo e a quantificação é substituída por algo ainda não-finitário, como os funcionais de tipo superior na demonstração analisada.

É razoável que o interesse na busca por demonstrações de consistência construtivas para sistemas como $P A$ persista apesar do segundo teorema da incompletude de Gödel, que impede demonstrações de consistência que sejam formalizáveis em tais sistemas. A análise dos casos acima mostra que eliminar quantificadores substituindo-os por algo cuja interpretação seja mais próxima do finitário é parte importante da estratégia de demonstração de consistência dentro do quadro delimitado por Gödel. As etapas do esquema geral proposto são cumpridas construtivamente apenas após alguma eliminação de quantificadores. Isso ajuda a ex- plicar a dificuldade de demonstrar construtivamente a consistência de um sistema como $Z F C$, para o qual parece muito difícil substituir a quantificação por algo mais próximo do finitário e mais facilmente interpretado. No entanto, $o$ esforço para avançar nas demonstrações construtivas de consistência frequentemente leva a um melhor entendimento sobre a estrutura quantificacional do sistema e sua interpretação.

Ainda, observamos que tais demonstrações, além do seu interesse fundacional, enriquecem a disciplina da lógica matemática enquanto método para atacar problemas matemáticos, tendo como fruto a área conhecida como teoria da demonstração. Como corolário da demonstração de consistência de $P A$ e da demonstração do Teorema de Herbrand temos, por exemplo, um resultado devido a Kreisel que estabelece que para bloquear potenciais contraexemplos para uma fórmula fechada em forma normal prenexa que é dedutível em $P A$ basta considerar os funcionais recursivos. Esse resultado já foi usado para extrair informação construtiva de demonstrações matemáticas nãoconstrutivas. Os recentes avanços desta área podem ser encontrados em Kohlenbach. 
5 - Considerações Finais Sobre Consistência e Verdade Aritmética

Podemos considerar a relação entre consistência e verdade na aritmética sob o prisma do estudo de casos acima. Em linhas gerais, dizemos de uma sentença declarativa que ela é verdadeira com relação a um padrão de correção se uma concordância entre a sentença e o padrão é obtida. Caso contrário, dizemos que a sentença é falsa com relação ao padrão de correção instituído. Nesse sentido, um discurso sobre verdade de sentenças da aritmética deve vir acompanhado de uma elucidação dos elementos centrais em torno dos quais esta concepção geral se articula. Logo, antes de falar em verdades aritméticas precisamos explicar o que é uma sentença (declarativa), que tipo de coisa é um padrão de correção, como um padrão é instituído e em que consiste a relação de concordância entre sentença e padrão.

Outros tipos de sentença podem ser considerados, mas vamos nos ocupar aqui apenas com o discurso sobre verdade de sentenças em uma linguagem de primeira ordem, como a linguagem de $P A$. De um modo geral, o problema de definir precisamente as sentenças de uma teoria matemática não oferece grande dificuldade. É muito mais difícil explicar satisfatoriamente que tipo de coisa é um padrão de correção em uma teoria matemática, como ele é instituído e em que consiste a relação de concordância entre sentença e padrão. Uma primeira tentativa seria a concepção de verdade como validade em uma classe de estruturas: Um padrão de correção em uma teoria matemática é uma classe de estruturas formada pelos modelos padrão da teoria; esse padrão é instituído pela intenção do matemático de falar dessas estruturas ao formular sua teoria, e uma sentença concorda com esse padrão se é satisfeita em todas as estruturas da classe.

Um esclarecimento deve ser feito sobre o que entendemos por estrutura, no nosso caso, estruturas da aritmética de primeira ordem. Uma tal estrutura é constituída por um domínio qualquer de indivíduos, uma relação binária (nesse domínio) que interpreta o símbolo de igualdade $=$, um indivíduo que interpreta o símbolo 0 e duas operações binárias que interpretam os símbolos,+ ., para adição e multiplicação. Reforçamos que não há restrição adicional para os dados de uma estrutura, ou seja, o domínio, a relação binária e as operações. É usual pedir que o número de indivíduos no domínio seja maior que zero e que a relação que interpreta igual- 
dade seja a relação de identidade no domínio, mas mesmo esses requerimentos podem ser dispensados. Claro que nem todas as estruturas são modelos padrão intencionados.

Dado o esclarecimento, ao retornar à tentativa, observamos os seguintes problemas. Não é claro que ao formular uma teoria a intenção do matemático de se dirigir aos modelos padrão é suficiente, ainda que esses modelos sejam entendidos por meio de uma metateoria. Não basta ter uma intenção para garantir que as sentenças da teoria adquiram um sentido específico. Estruturas que servem de base para a interpretação da linguagem são usualmente entendidas através de uma teoria matemática. Nesse caso, o entendimento proposto sobre verdade de sentenças em uma teoria matemática só poderia ser dado em outra teoria matemática, uma metateoria, capaz de definir tanto as fórmulas da teoria objeto quanto as estruturas apropriadas. Um discurso sobre verdade de sentenças aritméticas acaba por ser realizado em uma metateoria, portanto mediado por outra teoria matemática. Tal mediação é indesejada pois a aritmética é uma teoria matemática básica e parece natural que possamos entender sua verdade sem ter que recorrer à outra teoria.
Se tentamos resolver esse problema supondo que o entendimento de modelos padrão intencionados prescinde de uma metateoria, então a situação se agrava por outro lado. Pois teríamos agora dois tipos de entendimento dos modelos padrão, um mediado pela metateoria e outro direto. E assim, ou esse entendimento direto dos modelos padrão concorda com as representações teóricas destes, ou o conhecimento matemático sobre os modelos padrão estaria errado. Como a segunda alternativa não é admissível se temos como hipótese que o conhecimento matemático sobre os modelos não pode estar errado, temos que nos comprometer com a concordância entre o entendimento direto de modelos e o entendimento por meio de uma metateoria. Mas tal comprometimento parece inadequado e poderia trazer consequências posteriores, portanto preferimos buscar outra alternativa.

Portanto, vamos trabalhar em detalhes uma proposta alternativa para a relação de verdade na aritmética. A finalidade dessa nova proposta é eliminar a referência à classe de modelos padrão preservando o ideal almejado. Uma teoria matemática, segundo a proposta acima, dirige-se, através da intenção do matemático, à classe de modelos padrão, almejando, 
com isso, alcançar exatamente as sentenças satisfeitas em todos os modelos padrão. A chave para a formulação da nova proposta é o entendimento de que essa direção que a teoria possui, ou seja, a noção que a teoria almeja alcançar as sentenças satisfeitas em todos os modelos padrão, pode (i) ser caracterizada sem referência à classe de modelos padrão e (ii) desempenhar o papel de padrão de correção. Se isso for o caso, então os problemas mencionados acima não atingem a nova proposta, enquanto os méritos da concepção de verdade como validade em uma classe de estruturas são mantidos.

Há um problema sério com a tese segundo a qual uma classe de modelos padrão tem prioridade sobre o critério objetivo de seleção de estruturas correspondentes. Essa tese toma por base que pelo menos uma classe de modelos padrão de uma teoria matemática está determinada em primeiro lugar, e, que a axiomatização da teoria é uma articulação subordinada aos modelos padrão que é obtida na tentativa de descrevê-los. Isso pode ser plausível para algumas teorias como, por exemplo, a aritmética, em que há uma representação simples e precisa dos indivíduos, predicados e operações de um modelo padrão. Contudo, o mesmo não ocorre para outras teoria, como a teoria de conjuntos. Não há representação simples, que não assuma de antemão a própria teoria de conjuntos e que determina exaustivamente um modelo dessa teoria, nem é o caso que uma classe de modelos padrão da teoria de conjuntos está determinada (em uma metateoria) e antecede sua axiomatização. Como acreditamos ser importante alcançar um entendimento da noção de verdade que seja uniforme para as teorias matemáticas, esse problema deve ser levado em consideração aqui.

Partimos, então, da ideia que se uma teoria matemática dirige-se a uma classe de modelos padrão, então essa direção que a teoria possui pode $(i)$ ser caracterizada sem referência à classe de modelos padrão e (ii) desempenhar o papel de padrão de correção. A propriedade (i) pode ser entendida do seguinte modo: Cada classe de modelos padrão é caracterizada por um critério objetivo de seleção de estruturas que pode ser formulado como uma lista de princípios, os princípios diretivos correspondentes à classe dada. Ou seja, cada classe de modelos padrão pode ser vista como a classe das estruturas que estão em conformidade com um critério correspondente. Contudo, essa formulação sugere que a classe de modelos padrão conti- 
nua desempenhando o papel principal, enquanto os princípios diretivos são secundários. Nós vamos inverter a ordem de prioridade, dando aos princípios diretivos o papel principal. Assim não é preciso se comprometer ontologicamente com a classe de modelos padrão para apresentar o critério que deveria ser satisfeito por cada um deles, pois não precisamos de modelos padrão para prescrever o que seria um.

Consideramos, portanto, que uma lista de princípios diretivos tem prioridade sobre a classe de modelos padrão correspondente, e a axiomatização de uma teoria matemática deve ser guiada por tal lista. Um padrão de correção primário para uma teoria matemática é a lista de princípios diretivos correspondente, a classe de modelos padrão correspondente deve ser entendida como um padrão de correção secundário e apenas na medida em que representa os princípios. Esse padrão é instituído na prática histórica da matemática, na medida em que os princípios são adotados, implícita ou explicitamente, e regem essa prática.

Vamos apresentar agora uma lista de princípios diretivos para a aritmética e, em seguida, uma explicação do papel da mesma na aritmética. Essa lista de princípios constitui uma direção a ser se- guida pela teoria. Podemos dizer que a prática da aritmética está sujeita à adoção de princípios diretivos instituídos historicamente, como aqueles apresentados aqui, e que essa prática regida por princípios antecede a axiomatização.

1. Cada número é denotado por um único numeral, sendo este um objeto sintético obtido pela repetição, possivelmente nula, de um símbolo primitivo. Cada numeral denota um único número e o número zero é denotado pelo numeral nulo.

2. Dados dois numerais $s$ e $t$, a adição dos números denotados por $s$ e $t$ é denotada pelo numeral obtido pela repetição do símbolo primitivo determinada pela concatenação de $t \mathrm{e}$ $s$.

3. Dados dois numerais $s$ e $t$, a multiplicação dos números denotados por $s$ e $t$ é denotado pelo numeral obtido pela repetição de $s$ determinada por $t$, ou seja, por uma repetição de $s$ para cada ocorrência do símbolo primitivo em $t$.

A lista de princípios acima definitivamente não é uma descrição de um modelo padrão dos "números verdadeiros"; ela apenas prescreve a direção seguida pela aritmética, ou seja, o que essa teorização almeja descrever. Esta 
lista prescreve o que um domínio qualquer de objetos com operações deve obrigatoriamente satisfazer para poder ser considerado um modelo padrão da aritmética, uma estrutura que a aritmética almeja descrever. É importante ressaltar que não faz sentido falar em valor de verdade do critério prescrito por essa lista de princípios uma vez que esta lista não desempenha função descritiva para ser verdadeira ou falsa. Podemos apenas dizer que os princípios diretivos foram instituídos historicamente pela relevância que a investigação na direção apontada apresenta, e que, uma vez instituídos, prescrevem objetivamente a direção da aritmética.

Os axiomas da aritmética devem ser escolhidos de forma que o sistema dedutivo resultante seja uma aproximação, tão boa quanto possível, das sentenças verdadeiras de acordo com o padrão de correção dado pelos princípios diretivos. A intenção de satisfazer tal aproximação resulta em uma escolha de um sistema dedutivo que seja um correlato formal da lista de princípios. Acreditamos que este é o modo pelo qual chegamos aos axiomas do sistema dedutivo $P A$, mesmo que implicitamente. Para o caso do axioma esquema da indução é válida uma explicação adicional: Esse axioma é obtido a partir do primeiro princípio, na tentativa de descrever uma situação em que os números são todos denotados por numerais.

A lista de princípios não constitui um âmbito externo à aritmética, e sim faz parte da aritmética enquanto sistema teórico interpretado. A aritmética, assim compreendida, é constituída por duas camadas: A camada do critério dado pela lista de princípios, em que é dada a direção da teoria, e a camada dos sistemas formais para a aritmética, que deve estar em concordância com os princípios e que constitui o âmbito dedutivo associado. Uma sentença formal da aritmética se encontra na segunda camada definida acima, e dizemos que ela é verdadeira se descreve corretamente a direção prescrita pelos princípios dados na primeira camada. Essa noção de verdade aritmética pode ser analisada matematicamente, e o resultado da análise matemática corresponde com o esperado. Para um tratamento mais técnico e abrangente desta proposta veja Freire.

Um fato relevante sobre a noção de verdade aritmética que acabamos de estabelecer é que ela não implica a consistência dos sistemas formais correspondentes. Mesmo que para alguns sistemas a demonstração de consistência seja plausível, uma concepção de verdade de teorias matemáticas deve 
ser uniforme, como dissemos anteriormente, e se aplicar igualmente às teorias para as quais não temos tais demonstrações. Se o sistema formal $P A$ é inconsistente, então, em virtude do mesmo fato, o critério estabelecido pela lista de princípios é necessariamente vazio, e não aponta para direção alguma. Isso não ocorre com a concepção de verdade como satisfa- ção em uma estrutura padrão que prescinde de uma metateoria, porque se essa estrutura satisfaz $P A$, então $P A$ é consistente. Julgamos que essa característica da concepção de verdade é desejável, pois, tendo em vista as limitações para demonstrações de consistência expostas na seção anterior, faz sentido deixar em aberto a possibilidade da inconsistência.

\section{Referências}

BOOLOS, George, Burgess, John, Jeffrey, Richard, Computabilidade e Lógica, Tradução de Cezar Mortari, São Paulo: Editora Unesp, 2012. BOURBAKI, Nicolas, Théorie des Ensembles, Paris: Hermann, 1970.

FREIRE, Rodrigo, Interpretation and Truth in Set Theory, em Trends in Logic: Vol. 47, Contradictions, from Consistency to Inconsistency, Berlim: Springer, 2018.

HILBERT, David, On the Infinite, em Philosophy of Mathematics: Selected Readings, editado por Paul Benacerraf e Hilary Putnam, Segunda Edição, Cambridge University Press, 1983, pp. 183 - 201.

KLEENE, Stephen, Introduction to Metamathematics, Amsterdam: NorthHolland Publishing Company, 1952.

KOHLENBACH, Ulrich, Applied Proof Theory: Proof Interpretations and their Use in Mathematics, Berlim: Springer-Verlag, 2008.

RAGGIO, Andres, A Evolução da Noção de Sistema Axiomático, Philosophos - Revista de Filosofia, vol. 8, no. 1, 2003, pp. 95 - 119.

SHOENFIELD, Joseph, Mathematical Logic, Reading, Massachusetts: Addison-Wesley Publishing Company, 1967.

SMORYNSKI, Craig, The Incompleteness Theorems, em Handbook of Mathmatical Logic, editado por Jon Barwise, Amsterdam: NorthHolland, 1977, pp. 821 - 865.

TARSKI, Alfred, Mostowski, Andrzej, Robinson, Raphael, Undecidable Theories, Amsterdam: North-Holland Publishing Company, 1953. VIERO, Arno, Sistemas Axiomáticos Formalizados: A Questão da Desinterpretação e da Formalização da Axiomática, Campinas: Coleção CLE, 2011. 